\title{
CONVEXITY AND VARIATION DIMINISHING PROPERTY FOR BERNSTEIN POLYNOMIALS IN HIGHER DIMENSIONS
}

\author{
MAREK BEŚSA
}

\begin{abstract}
Faculty of Applied Physics and Mathematics, Technical University of Gdanisk Majakowskiego 11/12, 80-952 Gdańsk, Poland
\end{abstract}

\section{Introduction}

Let $f(x)$ be a real function defined in $Q:=[0,1]$. The Bernstein polynomial associated with $f$ is defined by

$$
B^{n}(f ; x):=\sum_{i=0}^{n} f_{i} \cdot\left(\begin{array}{l}
n \\
i
\end{array}\right) x^{i}(1-x)^{n-i}, \quad x \in Q,
$$

where $f_{i}:=f(i / n)$ for $i=0,1, \ldots, n$.

Joining $\left((i-1) / n, f_{i-1}\right)$ and $\left(i / n, f_{i}\right)$ by a line segment, for $i=1,2, \ldots, n$, we get a piecewise linear continuous function which is denoted by $\hat{f}_{n}(x)$ and called the Bézier polygon of $B^{n}(f ; x)$.

It is easy to show that:

(i) If $\hat{f}_{n}(x)$ is convex in $Q$ then so is $B^{n}(f ; x)$.

(ii) If $f(x)$ is convex in $Q$ then

$$
B^{n}(f ; x) \geqslant B^{n+1}(f ; x), \quad n=1,2, \ldots, \quad \text { for } x \in Q .
$$

(iii) The variation diminishing property holds, i.e.

$$
V\left[B^{n} ; Q\right] \leqslant V_{1}\left[\hat{f}_{n} ; Q\right],
$$

with equality if and only if $\hat{f}_{n}(x)$ is convex or concave. Here

$$
V\left[B^{n} ; Q\right]:=\int_{Q}\left|\frac{d^{2} B^{n}}{d x^{2}}\right| d x,
$$

$$
V_{1}\left[\hat{f}_{n} ; Q\right]:=n \sum_{i=0}^{n-2}\left|\Delta^{2} f_{i}\right|
$$

$\Delta f_{i}:=f_{i+1}-f_{i}, \quad i=0,1, \ldots, n-1$. 
G. Chang, P. Davis and J. Hoschek (see [1], [3]) extended these results to the Bernstein polynomials over triangles. In the present paper we attempt to extend these results to the Bernstein polynomials over a $k$-dimensional simplex $Q$. In this case the definition of a variation like (5) called now the variation of the Bézier net is more complicated, but in the case of $k=1,2$ it coincides with the variations as given in [2], [3]. We also give an answer to the problem whether the variation of the Bézier net is equal to $\operatorname{Var}\left(\Delta f_{n}\right)$. Here the Laplace operator $\Delta$ is taken in the distribution sense (see a remark in Section 3). These problems were suggested to me by Professor Z. Ciesielski.

\section{Notation}

Let us begin with some definitions. Let $Q:=\left[P_{1}, P_{2}, \ldots, P_{k+1}\right]$ be the $k$-dimensional simplex with vertices $P_{1}, P_{2}, \ldots, P_{k+1}$, where $P_{i} \in R^{k}$ for $i=1,2, \ldots, k+1$. By $Q_{\alpha}(1 \leqslant \alpha \leqslant k+1)$ we denote the $(k-1)$-dimensional face of the simplex $Q$ which does not contain the vertex $P_{\alpha}$. By $W:=\left[T_{1}, T_{2}, \ldots, T_{k+1}\right]$ we denote the simplex with the vertices $T_{i}=(0, \ldots, 0,1,1, \ldots, 1)$, where $i=1,2, \ldots, k+1$, and by $K_{n}(W)$ the subdivision of the simplex $W$ such that a simplex $\Omega$ belongs to $K_{n}(W)$ if and only if

$$
\Omega=\Delta_{i_{1} i_{2} \ldots i_{k}}^{n}+v
$$

where

$$
\Delta_{i_{1} i_{2} \ldots i_{k}}^{n}=\left\{\left(x_{1}, \ldots, x_{k}\right) \in R^{k} ; 0 \leqslant x_{i_{1}} \leqslant x_{i_{2}} \leqslant \ldots \leqslant \bar{x}_{i_{k}} \leqslant 1 / n\right\},
$$

$i_{1}, i_{2}, \ldots, i_{k}$ is a permutation of $\{1,2, \ldots, k\}$, and the coordinates of the vector $v=\left(v_{1}, v_{2}, \ldots, v_{k}\right) \in R^{k}$ satisfy the following conditions:

(i) $n v_{i} \in N=\{0,1,2, \ldots\}$ for $i=1,2, \ldots, k$,

(ii) $0 \leqslant v_{1} \leqslant v_{2} \leqslant \ldots \leqslant v_{k^{k}}<1$.

Now, let $L: R^{k} \rightarrow R^{k}$ be an affine transformation such that

$$
L\left(T_{i}\right)=P_{i} \quad \text { for } i=1,2, \ldots, k+1 .
$$

Then $L$ transforms the subdivision $K_{n}(W)$ of the simplex $W$ to some subdivision of the simplex $Q$, which we denote by $S_{n}(Q)$. It is known that each point $P \in Q$ can be uniquely expressed as

$$
P=\sum_{i=1}^{k+1} u_{i} P_{i}
$$

with $u_{1}+u_{2}+\ldots+u_{k+1}=1, u_{i} \geqslant 0,1 \leqslant i \leqslant k+1$.

The numbers $u_{1}, u_{2}, \ldots, u_{k+1}$ are called the barycentric coordinates of $P$ with respect to the simplex $Q$. We identify the point $P \in Q$ with its barycentric coordinates and write $P=\left(u_{1}, u_{2}, \ldots, u_{k+1}\right)$. For $n \in N$ and a given 
function $f: Q \rightarrow R$ we define

$$
F_{n}:=\left\{f_{\beta} ; f_{\beta}:=f\left(\frac{\beta}{n}\right), \beta \in N^{k+1},|\beta|=n\right\}
$$

where

$$
\beta=\left(\beta_{1}, \beta_{2}, \ldots, \beta_{k+1}\right) \text { and }|\beta|:=\sum_{i=1}^{k+1} \beta_{i} .
$$

We also define

$$
B^{n}(f ; P)=\sum_{|\beta|=n} f_{\beta} J_{\beta}^{n}(P) \quad \text { for } P \in Q
$$

where

$$
J_{\beta}^{n}(P):=\frac{n !}{\beta !} u^{\beta}:=\frac{n !}{\beta_{1} ! \beta_{2} ! \ldots \beta_{k+1} !} u_{1}^{\beta_{1}} u_{2}^{\beta_{2}} \ldots u_{k+1}^{\beta_{k+1}}
$$

and $P=\left(u_{1}, u_{2}, \ldots, u_{k+1}\right)$.

The polynomials $J_{\beta}^{n}(P)$ are called the basic Bernstein polynomials. $B^{n}(f ; P)$ is called the $n$-th Bernstein polynomial over the simplex $Q$. Setting

$$
\tilde{P}_{\beta}:=\left(P, f_{\beta}\right):=\left(\frac{\beta_{1}}{n}, \frac{\beta_{2}}{n}, \ldots, \frac{\beta_{k+1}}{n}, f_{\beta}\right),
$$

we get a point on the surface associated with the function $f(P)$. The points $\tilde{P}_{\beta}$ with $|\beta|=n$ are called the Bézier points of $B^{n}(f ; P)$. For any simplex $\Omega \in S_{n}(Q)$ we have $k+1$ Bézier points. Joining them by a $k$-dimensional plane we obtain a continuous piecewise linear surface $\hat{f}_{n}(P)$, which is called the Bézier net of $B^{n}(f ; P)$ over the simplex $Q$.

Let $E_{i}(1 \leqslant i \leqslant k+1)$ be the partial shift operator defined by

$$
E_{i} f_{\beta}:=f_{\beta}+\hat{e}_{i}
$$

where $|\beta|=n-1, \hat{e}_{i}=(0, \ldots, 0,1,0, \ldots, 0) \in R^{k+1}$.

For convenience we introduce

$$
D_{i j}\left(f_{\beta}\right):=-\left(E_{i}-E_{i+1}\right)\left(E_{j}-E_{j+1}\right) f_{\beta}
$$

where $|\beta|=n-2,1 \leqslant i<j \leqslant k+1$ and $E_{k+2}:=E_{1}$.

\section{Results}

The first theorem generalizes a result of G. Chang and P. Davis [1].

THEOREM 1. The convexity of the Bézier net $\hat{f}_{n}(P)$ over $Q$ is equivalent to the following inequalities:

$$
D_{i j}\left(f_{\beta}\right) \geqslant 0
$$

for $1 \leqslant i<j \leqslant k+1,|\beta|=n-2$. 
The proof is standard and will be omitted.

The next theorem is also a multidimensional analogue of a.theorem of G. Chang and P. Davis.

Theorem 2. (i) If the Bézier net $\hat{f}_{n}(P)$ over $Q$ is convex then so is the Bernstein polynomial $B^{n}(f ; P)$.

(ii) If $f(P)$ is convex in $Q$ then we have

$$
B^{n}(f ; P) \geqslant B^{n+1}(f ; P) \quad \text { for } P \in Q, n=1,2, \ldots
$$

Proof. (i) The proof is based on the well-known convexity test for any function in $C^{2}(Q)$ and the following

Lemma 1. For the Bernstein polynomial $B^{n}(f ; P)$ we have the equality

$$
\begin{aligned}
\frac{\partial^{2} B^{n}(f ; P)}{\partial x_{r} \partial x_{r^{\prime}}}= & \frac{n(n-1)}{k^{2}|Q|} \sum_{|\beta|=n-2} \sum_{1 \leqslant i<j \leqslant k+1}\left(\sum_{\alpha=i+1}^{j} n_{\alpha}\left|Q_{\alpha}\right|, e_{r}\right) \\
& \times\left(\sum_{\alpha=i+1}^{j} n_{\alpha}\left|Q_{\alpha}\right|, e_{r^{\prime}}\right) D_{i j}\left(f_{\beta}\right) J_{\beta}^{n}(P) \quad \text { for } r, r^{\prime}=1,2, \ldots, k
\end{aligned}
$$

where $P \in Q, \quad e_{r}=(0, \ldots, 0, \dot{i}, 0, \ldots, 0) \in R^{k}, \quad r=1,2, \ldots, k,|Q|:=\operatorname{vol}_{k} Q$, $\left|Q_{\alpha}\right|:=\operatorname{vol}_{k-1}\left(Q_{\alpha}\right),(\cdot, \cdot)$ is the scalar product in $R^{k}$, and $n_{\alpha}$ is the unit outward normal vector to $Q_{\alpha}$.

The tedious proof will be omitted.

(ii) G. Chang and P. Davis ([1]) proved the statement for $k=2$. For $k>2$ the proof is similar.

Now, we define a variation of the Bernstein polynomial $B^{n}(f ; P)$ by

$$
V\left[B^{n} ; Q\right]:=\int_{Q}\left|\Delta B^{n}\right|
$$

where for simplicity $B^{n}$ stands for $B^{n}(f ; P)$ and $\Delta$ is the Laplacian. The variation $V_{1}\left[\hat{f}_{n}, Q\right]$ of the Bézier net $\hat{f}_{n}(P)$ is defined by

$$
V_{1}\left[\hat{f}_{n} ; Q\right]:=\frac{n !(k-1) !}{k(n+k-2) !|Q|} \sum_{|\beta|=n-2} \sum_{1 \leqslant i<j \leqslant k+1}\left|D_{i j}\left(f_{\beta}\right)\right| \|\left.\sum_{\alpha=i+1}^{j} n_{\alpha}\left|Q_{\alpha}\right|\right|^{2}
$$

where $\|\cdot\|^{2}=(\cdot, \cdot)$.

For $k=2$ the variation $V_{1}\left[\hat{f}_{n} ; Q\right]$ of the Bézier net coincides with the variation introduced by $T$. Goodman [3]. Our next theorem generalizes the result obtained by $G$. Chang and J. Hoschek [2].

TheOREм 3. For the Bernstein polynomial over the simplex $Q$ the variation diminishing property holds. More precisely, we have

$$
V\left[B^{n} ; Q\right] \leqslant V_{1}\left[\hat{f}_{n} ; Q\right]
$$

with equality if and only if $\hat{f}_{n}(P)$ is either convex or concave. 
For $k=1,2$ the theorem was proved by G. Chang and J. Hoschek [2]. For $k>2$ the proof is similar.

Since

$$
V\left[B^{n} ; Q\right]=\operatorname{Var}\left(\Delta B^{n}\right)
$$

where $\operatorname{Var}\left(\Delta B^{\eta}\right)$ is the total variation of the measure

$$
v(A):=\int_{A} \Delta B^{n}
$$

the question arises whether the equality

$$
V_{1}\left[\hat{f}_{n} ; Q\right]=\operatorname{Var}\left(\Delta \hat{f}_{n}\right)
$$

holds.

Remark. Here the Laplace operator $\Delta$ is taken in the distribution sense and because $\Delta \hat{f}_{n}$ is a measure (not necessarily non-negative), the total variation $\operatorname{Var}\left(\Delta \hat{f}_{n}\right)$ is well defined.

The following lemma solves this problem for $k=1,2$.

LeMma 2. For $k=1,2$ the equality (16) holds.

Proof. For $k=1$ the proof is very simple.

If $k=2$ the simplex $Q=\left[P_{1}, P_{2}, P_{3}\right]$ with vertices $P_{1}=P_{1}\left(x_{1}, y_{1}\right), P_{2}$ $=P_{2}\left(x_{2}, y_{2}\right), P_{3}=P_{3}\left(x_{3}, y_{3}\right)$ is a triangle. Without loss of generality we can assume that

$$
\operatorname{det}\left[P_{1}-P_{3}, P_{2}-P_{3}\right]:=\operatorname{det}\left[\begin{array}{ll}
x_{1}-x_{3} & x_{2}-x_{3} \\
y_{1}-y_{3} & y_{2}-y_{3}
\end{array}\right]>0 \text {. }
$$

The Stokes theorem yields

$$
\begin{aligned}
\left(\Delta \hat{f}_{n}\right)(\varphi) & =\int_{Q} \hat{f}_{n} \Delta \varphi \\
& =-\sum_{\Omega \in S_{n}(Q)}\left(\int_{\partial \Omega} \frac{\partial \hat{f}_{n}}{\partial x} m_{1} \varphi d \sigma+\int_{\partial \Omega} \frac{\partial \hat{f}_{n}}{\partial y} m_{2} \varphi d \sigma\right) \\
& =-\sum_{\Omega \in S_{n}(Q)} \sum_{r=1}^{3}\left(\int_{\Omega} \frac{\partial \hat{f}_{n}}{\partial x} m_{1} \varphi d \sigma+\int_{\Omega} \frac{\partial \hat{f}_{n}}{\partial y} m_{2} \varphi d \sigma\right)
\end{aligned}
$$

where $\varphi \in D($ Int $Q)$, the space of test functions, $\left(m_{1}, m_{2}\right)$ is the unit outward normal vector to the boundary $\partial \Omega$, and $\sigma$ is the Lebesgue measure on $\partial \Omega$. First we consider the sum

$$
\sum_{\Omega \in S_{n}(Q)} \int_{\Omega_{1}} \frac{\partial \hat{f}_{n}}{\partial x} m_{1} \varphi d \sigma
$$

If $\Omega_{1} \not \subset$ (for $\left.\Omega \in S_{n}(Q)\right)$ we can find a second triangle $\Omega^{\prime}$ such that $\Omega_{1}=\Omega_{1}^{\prime}$. 
Thus the sum (18) is equal to

$$
\sum_{\substack{\Omega=S_{n}(Q) \\ \Omega_{1} \notin Q_{1}}}\left(\int_{\Omega_{1}} \frac{\partial \hat{f}_{n}}{\partial x} m_{1} \varphi d \sigma+\int_{\Omega_{1}^{\prime}} \frac{\partial \hat{f}_{n}}{\partial x} m_{1}^{\prime} \varphi d \sigma\right)
$$

Since $\left(m_{1}^{\prime}, m_{2}^{\prime}\right)=-\left(m_{1}, m_{2}\right)$ the expression (19) is equal to

$$
\frac{1}{2} \sum_{\substack{\Omega \in S_{n}(Q) \\ \Omega_{1} \neq Q_{1}}}\left(\frac{\left.\partial \hat{f}_{n}\right|_{\Omega}}{\partial x}-\frac{\left.\partial \hat{f}_{n}\right|_{\Omega_{m_{1}}^{\prime}}}{\partial x}\right) \int_{\Omega_{1}} \varphi d \sigma
$$

where $\left.\hat{f}_{n}\right|_{\Omega},\left.\hat{f}_{n}\right|_{\Omega}$ are the restrictions of $\hat{f}_{n}$ to $\Omega^{\prime}, \Omega$, respectively. We can assume that the barycentric coordinates of the vertices of the triangles $\Omega$ $=\left[U_{1}, U_{2}, U_{3}\right], \Omega^{\prime}=\left[U_{1}^{\prime}, U_{2}^{\prime}, U_{3}^{\prime}\right] \in S_{n}(Q)\left(\Omega_{1} \notin Q_{1}, \Omega_{1}=\Omega_{1}^{\prime}\right)$ are the following:

$$
\begin{gathered}
U_{1}=\left(\frac{i}{n}, \frac{j}{n}, \frac{k}{n}\right), \quad U_{2}=\left(\frac{i-1}{n}, \frac{j+1}{n}, \frac{k}{n}\right), \quad U_{3}=\left(\frac{i-1}{n}, \frac{j}{n}, \frac{k+1}{n}\right), \\
U_{1}^{\prime}=\left(\frac{i-2}{n}, \frac{j+1}{n}, \frac{k+1}{n}\right), \quad U_{2}^{\prime}=\left(\frac{i-1}{n}, \frac{j+1}{n}, \frac{k}{n}\right), \quad U_{3}^{\prime}=\left(\frac{i-1}{n}, \frac{j}{n}, \frac{k+1}{n}\right),
\end{gathered}
$$

for $i+j+k=n, j, k \geqslant 0, i \geqslant 2$.

Now by elementary calculations we get

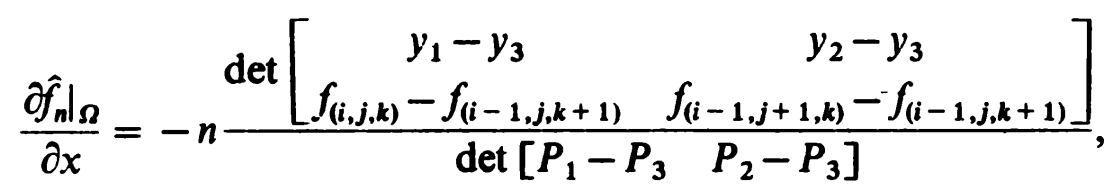

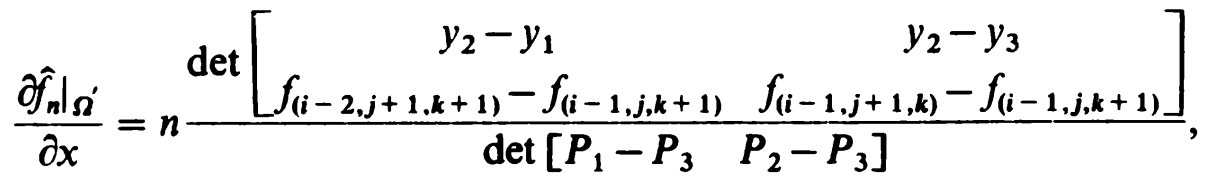

$$
m_{1}=\frac{y_{3}-y_{2}}{\left|Q_{1}\right|}, \quad m_{2}=\frac{x_{2}-x_{3}}{\left|Q_{1}\right|} .
$$

Thus the sum $(20)$ is equal to

(24) $\sum_{\substack{i+j+k=n \\ j, k \geqslant 0 \\ i \geqslant 2}} \frac{n\left(y_{2}-y_{3}\right)^{2}}{2|Q|\left|Q_{1}\right|}\left(f_{(i-1, j+1, k)}+f_{(i-1, j, k+1)}-f_{(i, j, k)}-f_{(i-2, j+1, k+1)}\right) \int_{\Omega_{1}} \varphi d \sigma$

$$
\begin{aligned}
& =-\sum_{\substack{i+j+k=n \\
j, k \geqslant 0 \\
i \geqslant 2}} \frac{n\left(y_{2}-y_{3}\right)^{2}}{2|Q|\left|Q_{1}\right|} D_{13} f_{(i-2, j, k)} \int_{\Omega_{1}} \varphi d \sigma \\
& =-\sum_{\substack{i+j+k=n-2 \\
i, j, k \geqslant 0}} \frac{n\left(y_{2}-y_{3}\right)^{2}}{2|Q|\left|Q_{1}\right|} D_{13} f_{(i, j, k)} \int_{\Omega_{1}} \varphi d \sigma .
\end{aligned}
$$


In a similar way we obtain

$$
\sum_{\Omega \in S_{n}(Q)} \int_{\Omega_{1}} \frac{\partial \hat{f}_{n}}{\partial y} m_{2} \varphi d \sigma=-\sum_{i+j+k=n-2} \frac{n\left(x_{2}-x_{3}\right)^{2}}{2|Q|\left|Q_{1}\right|} D_{13} f_{(i, j, k)} \int_{\Omega_{1}} \varphi d \sigma .
$$

Hence

(26) $\sum_{\Omega \in S_{n}(Q)} \int_{\Omega_{1}}\left(\frac{\partial \hat{f}_{n}}{\partial x} m_{1}+\frac{\partial \hat{f}_{n}}{\partial y} m_{2}\right) \varphi d \sigma=-\sum_{i+j+k=n-2} \frac{n\left|Q_{1}\right|}{2|Q|} D_{13} f_{(i, j, k)} \int_{\Omega_{1}} \varphi d \sigma$.

Now it is easy to check that

(27) $\sum_{\Omega \in S_{n}(Q)} \int_{\Omega_{2}}\left(\frac{\partial \hat{f}_{n}}{\partial x} m_{1}+\frac{\partial \hat{f}_{n}}{\partial y} m_{2}\right) \varphi d \sigma=-\sum_{i+j+k=n-2} \frac{n\left|Q_{2}\right|}{2|Q|} D_{12} f_{(i, j, k)} \int_{\Omega_{2}} \varphi d \sigma$,

(28) $\sum_{\Omega_{\in} S_{n}(Q)} \int_{\Omega_{3}}\left(\frac{\partial \hat{f}_{n}}{\partial x} m_{1}+\frac{\partial \hat{f}_{n}}{\partial y} m_{2}\right) \varphi d \sigma=-\sum_{i+j+k=n-2} \frac{n\left|Q_{3}\right|}{2|Q|} D_{23} f_{(i, j, k)} \int_{\Omega_{3}} \varphi d \sigma$.

By (26), (27), (28) the variation of $f_{n}$ is equal to

$$
\begin{aligned}
& \operatorname{Var}\left(\Delta \hat{f}_{n}\right) \\
& =\frac{1}{2|Q|} \sum_{i+j+k=n-2}\left(\left|Q_{1}\right|^{2}\left|D_{13} f_{(i, j, k)}\right|+\left|Q_{2}\right|^{2}\left|D_{12} f_{(i, j, k)}\right|+\left|Q_{3}\right|^{2}\left|D_{23} f_{(i, j, k)}\right|\right) \\
& =V_{1}\left[\hat{f}_{n} ; Q\right] .
\end{aligned}
$$

In case $k>2$ equality (16) does not hold. More precisely the two variations $\operatorname{Var}\left(\Delta \hat{f}_{n}\right), V_{1}\left[\hat{f}_{n} ; Q\right]$ are incomparable.

EXAMPLE 1. Let

$$
f_{\beta}=\left\{\begin{array}{ll}
1 & \text { for } \beta=n \hat{e}_{i}, \\
0 & \text { for } \beta \neq n \hat{e}_{i},
\end{array} \quad i=1,2, \ldots, k+1,|\beta|=n .\right.
$$

One can prove that the following equalities hold:

$$
\begin{gathered}
V_{1}\left[f_{n} ; Q\right]=\frac{n !(k-1) !}{k|Q|(n+k-2) !} \sum_{j=1}^{k+1}\left|D_{j, j+1}\left(f_{\beta_{j+1}}\right)\right| \cdot\left|Q_{j+1}\right|^{2}, \\
\operatorname{Var}\left(\Delta \hat{f}_{n}\right)=\frac{1}{k|Q| n^{k-2}} \sum_{j=1}^{k+1}\left|D_{j, j+1}\left(f_{\beta_{j+1}}\right)\right| \cdot\left|Q_{j+1}\right|^{2},
\end{gathered}
$$

where $\beta_{j}=(n-2) \hat{e}_{j}$ for $j=1,2, \ldots, k+1$ and $D_{k+1, k+2}:=D_{1, k+1}, \beta_{k+2}$ : $=\beta_{1}, Q_{k+2}:=Q_{1}$.

Now, it is easy to see that

$$
V_{1}\left[\hat{f}_{n}, Q\right]>\operatorname{Var}\left(\Delta \hat{f}_{n}\right) \quad \text { for } k=3,4, \ldots, n=2,3, \ldots
$$


Because the Bézier net is convex (by Theorem 1) we obtain (by Theorem 3)

$$
\operatorname{Var}\left(\Delta B^{\eta}\right)=V_{1}\left[\hat{f}_{n} ; Q\right]>\operatorname{Var}\left(\Delta \hat{f}_{n}\right) \text {. }
$$

Hence, the multidimensional version of the variation diminishing property for the Laplacian does not hold for $k>2$.

Example 2. Let $n=p(k+1)+2, k, p \in N, p \geqslant 3$. Define

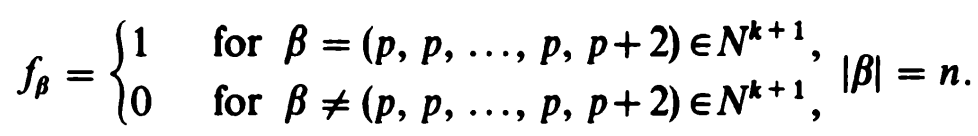

One can prove that the following equalities hold

$$
\begin{gathered}
V_{1}\left[\hat{f}_{n} ; Q\right]=\frac{n !(k-1) !}{k|Q|(n+k-2) !} \sum_{|\beta|=n-2} \sum_{1 \leqslant i<j \leqslant k+1}\left|D_{i j}\left(f_{\beta}\right)\right|\left\|\sum_{\alpha=i+1}^{j} n_{\alpha}\left|Q_{\alpha}\right|\right\|^{2}, \\
\operatorname{Var}\left(\Delta \hat{f}_{n}\right)=\frac{(k-1) !}{k|Q| n^{k-2}} \sum_{|\beta|=n-2} \sum_{1 \leqslant i<j \leqslant k+1}\left|D_{i j}\left(f_{\beta}\right)\right|\left\|\sum_{\alpha=i+1}^{j} n_{\alpha}\left|Q_{\alpha}\right|\right\|^{2}
\end{gathered}
$$

Now, it is easy to see that

$$
V_{1}\left[\hat{f}_{n} ; Q\right]<\operatorname{Var}\left(\Delta \hat{f}_{n}\right) \quad \text { for } n=2,3, \ldots, k=3,4, \ldots
$$

Fortunately, we can estimate the variation $\operatorname{Var}\left(\Delta \hat{f}_{n}\right)$ by $V_{1}\left[\hat{f}_{n} ; Q\right]$.

Lemma 3. For the Bézier net $\hat{f}_{n}$ we have the inequalities

$$
\frac{(n+k-2) !}{n^{k-2} n !} V_{1}\left[\hat{f}_{n} ; Q\right] \geqslant \operatorname{Var}\left(\Delta \hat{f}_{n}\right) \geqslant \frac{1}{(k-1) !} V_{1}\left[\hat{f}_{n} ; Q\right],
$$

$k=1,2, \ldots, n=2,3, \ldots$

The tedious proof will be omitted.

Theorem 3 and the above lemma yield the "multidimensional version of the variation diminishing property for the Laplacian".

TheOREM 4. For the Bernstein polynomial $B_{n}(f ; P)$ over the $k$-dimensional simplex $Q$ the following inequality holds:

$$
\operatorname{Var}\left(\Delta B^{n}\right) \leqslant(k-1) ! \operatorname{Var}\left(\Delta \hat{f}_{n}\right),
$$

where for large $n$ the constant $(k-1)$ ! is best possible.

Acknowledgement. I would like to thank Professor Z. Ciesielski for his valuable comments and suggestions. 


\section{References}

[1] G. Chang and P. Davis, The convexity of Bernstein polynomials over triangles, J. Approx. Theory 40 (1984), 11-28.

[2] G. Chang and J. Hoschek, Convexity and variation diminishing property of Bernstein polynomials over triangles. Intern. Series Numer. Math. vol. 75, 1985, Multivariate Approx. Theory III.

[3] T. Goodman, Variation Diminishing Properties of Bernstein Polynomials on Triangles, Preprint, Department of Mathematical Sciences, The University of Dundee, Scotland.

Presented to the Semester

Approximation and Function Spaces

February 27-May 27, 1986 